Kommentar: Übereinstimmend mit den Befunden bei Adoleszenten zeigt die Studie, dass längere Wochenarbeitszeit mit Alkoholproblemen assoziiert ist. Dieser Zusammenhang zeigt keinen Geschlechtsunterschied. Das ist unerwartet, weil Männer bis zu viermal häufiger unter Alkoholabhängigkeit leiden als Frauen.

Aufgrund des Studiendesigns kann trotz der umfangreichen Bemühungen zur Kon- trolle einer Vielzahl von beeinflussenden Faktoren kein kausaler Zusammenhang abgeleitet werden. Vielmehr diskutieren die Autoren zudem, dass erhöhter Stress und mehr soziale Kontakte während längerer Arbeitszeiten für den erhöhten Alkoholkonsum verantwortlich sein könnten. Einschränkend sei auch erwähnt, dass es sich um eine neuseeländische Geburtskohorte aus dem Jahr 1977 handelt, deren Ergebnis- se nicht ohne weiteres übertragen werden können.

Die Befunde könnten zukünftig hilfreich bei der Entwicklung von Maßnahmen zur Prävention und Detektion von Risikopersonen sein.

PD Dr. med. Bernd Lenz

Gibb SJ, et al. Working hours and alcohol problems in early adulthood. Addiction 2012; 107 (1): $81-8$

\title{
Naltrexon verhilft Rauchern zu einem günstigeren Verlauf einer komorbiden Alkoholkrankheit
}

\begin{abstract}
Allgemein haben Raucher eine schlechtere Prognose ihrer Alkoholkrankheit und schlechteres Ansprechen auf eine Therapie als Nichtraucher. Eine aktuelle Studie zeigt erstmalig, dass rauchende Alkoholkranke besonders von der Therapie mit Naltrexon profitieren.
\end{abstract}

Die ie Kombination von Alkohol- und Nikotinabhängigkeit stellt ein gravierendes gesellschaftliches Problem dar. $45 \%$ der Alkoholabhängigen sind Raucher. Sie verspüren gesteigerten Trinkdrang, erleben häufiger Rezidive mit anschließend vermehrtem Alkoholkonsum und zeigen ein schlechteres therapeutisches Ansprechen als Nichtraucher.

Gemäß der Datenlage reduziert Naltrexon bei Gelegenheitstrinkern das übermäßige Trinkverhalten. Der Opioidrezeptorantagonist mildert das Schlüsselreiz-induzierte Verlangen sowie die Höhe des Alkoholkonsums nach einem Rückfall.

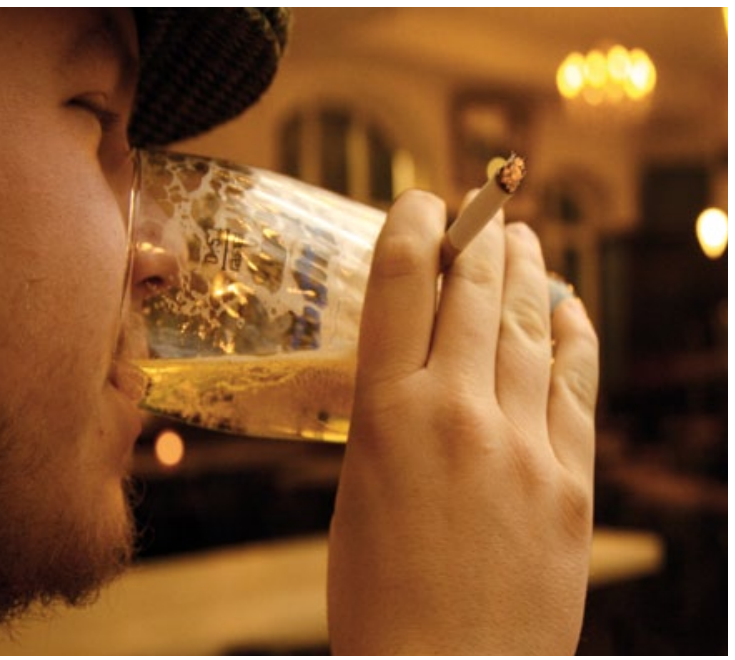

Fatale Kombination: Alkohol und Rauchen
Bei Alkoholkranken mit dem Wunsch, ihren Alkoholkonsum zu reduzieren oder zu beenden, untersuchten Fucito et al. den Behandlungseffekt von Naltrexon in Abhängigkeit davon, ob die betroffenen Alkoholkranken rauchten oder nicht. Der Datensatz stammt aus der weltweit größten, randomisierten, doppelblinden, placebokontrollierten Alkoholismusstudie COMBINE („Combines Pharmacotherapies and Behavioral Interventions for Alcohol Dependence"). Bei 1.226 Studienteilnehmern wurde mithilfe linearer Regressionsmodelle geprüft, inwiefern das Rauchverhalten zum Ausgangszeitpunkt und die erhaltene Medikation (Naltrexon 100 $\mathrm{mg} / \mathrm{d}$ oder Placebo) das Trink- sowie Rauchverhalten nach 16-wöchiger Therapie beeinflussen.

Der potenzielle Zusammenhang zwischen Rauchverhalten und Erfolg der Naltrexonbehandlung zeigte sich in Bezug auf die Abstinenztage (bei alkoholabhängigen Rauchern 78,36 Tage unter Naltrexonbehandlung vs. 71,65 Tage ohne Naltrexonbehandlung; $p=0,004$ ) sowie die trinkbezogenen Folgen (bei alkoholabhängigen Rauchern 13,57 Konsequenzen unter Naltrexonbehandlung vs. 17,50 Konsequenzen ohne Naltrexonbehandlung; $p=0,01$ ). Letztere wurden zum Ausgangszeitpunkt, nach 8 und 16 Wochen mit dem „Drinker Inventory of Consequences" (Miller et al., 1995) erfasst. Bei alkoholkranken Nichtrau- chern zeigte sich keine Überlegenheit von Naltrexon gegenüber Placebo.

Alkoholabhängige Raucher unter Naltrexon-Behandlung können also im Vergleich zur Placebogruppe länger abstinent bleiben und erleiden weniger trinkbezogene Folgeschäden. Einen Effekt auf das Rauchverhalten erzielte Naltrexon nicht .

Kommentar: Die vorliegende Studie ergänzt die Literatur über Prädiktoren der Wirksamkeit von Naltrexon in der Therapie der Alkoholkrankheit. Rauchen als prädiktive Variable ist im klinischen Alltag besser umsetzbar als eine zeit- und kostenintensive Phäno- und Genotypisierung.

Einschränkend ist zu erwähnen, dass Fucito et al. eine Naltrexon-Dosierung verwendeten, die höher ist als die standardmäßig in der Alkoholabhängigkeitstherapie eingesetzte Dosis. Da sich eine Reduktion des Zigarettenkonsums positiv auf das Trinkverhalten auswirkt, sollte man gezielter evidenzbasierte Interventionen zur Raucherentwöhnung einsetzen. Zusammenfassend zeigen die Studienergebnisse die Relevanz des Rauchens als Prädiktor für das therapeutische Ansprechen alkoholabhängiger Patienten.

Dr. med. Birgit Braun

Fucito LM, et al. Cigarette smoking predicts differential benefit from naltrexone for alcohol dependence. Biol Psychiatry. 2012; 72 (10): 832-8 